\title{
Operations and Performance of the CMS DT and RPC muon systems
}

\author{
Gabriella Pugliese* \\ On behalf of the CMS Collaboration \\ Dipartimento Interateneo di Fisica and Sezione INFN, Via G. Amendola 173, 70126 Bari, Italy \\ E-mail: gabriella.pugliese@ba.infn.it
}

The muon spectrometer of the Compact Muon Solenoid (CMS) at the CERN Large Hadron Collider (LHC) is equipped with a redundant muon system based on Drift Tubes (DT) Chambers, Cathode Strip Chambers (CSC) and Resistive Plate Chambers (RPC) for muon identification, precise momentum measurement and triggering. On 30th March 2010, LHC started proton proton collisions at a center of mass energy of $7 \mathrm{TeV}$. The operations and performance of the DT and RPC systems during the first three years of LHC activity with increasing instantaneous luminosity will be reported. The DT local trigger performance is described, showing how the challenging design goals have been met. Special attention will be given to the RPC working point calibration procedure and to the efficiency stability of the RPC system, confirming the excellent behavior and the fulfillment of the CMS requirements. Finally the radiation background levels in the DT and RPC systems have been measured. Extrapolations to the LHC design conditions are also discussed.

36th International Conference on High Energy Physics

4-11 July 2012

Melbourne, Australia

${ }^{*}$ Speaker. 


\section{Introduction}

The Compact Muon Solenoid (CMS) [1] is a multi-purpose particle physics detector built at the CERN Large Hadron Collider (LHC) [2]. The CMS basic design element is a super-conducting solenoid that produces a $3.8 \mathrm{~T}$ magnetic field: the tracker, the electromagnetic and hadron calorimeters are within the field volume; while the iron yoke is instrumented with a muon spectrometer for muon identification, momentum measurement and triggering. The $\mu$ spectrometer is divided into 5 separate wheels in the barrel (named $\mathrm{YB} \pm 2, \mathrm{YB} \pm 1$ and $\mathrm{YB} 0$ ) and 4 independent disks both in the positive and negative endcaps (named $\mathrm{YE} \pm 4, \mathrm{YE} \pm 3, \mathrm{YE} \pm 2, \mathrm{YE} \pm 1$ ). The 4 th disk will be installed during the LHC long shutdown in 2013-2014. Three different gaseous detector technologies are employed: Drift Tube (DT) chambers in the barrel region to detect muons up to pseudo-rapidity $|\eta|<1.2$; Cathode Strip Chambers (CSCs) to handle the higher rates and non-uniform magnetic field in the endcap region $0.9<|\eta|<2.4$; Resistive Plate Chambers (RPCs) located in both barrel and endcap regions, up to $|\eta|<1.6$. Each wheel is divided into 12 sectors covering the full azimuthal angle $(\phi)$; each sector consists of four muon stations of DTs (named MB1 $\div 4$ ) coupled with RPCs (named RB $1 \div 4$ ). A DT chamber is made of three (or two in MB4) Super-layers (SL), each consisting of four layers of rectangular drift cells staggered by half tube width. The wires in the inner and outer SLs are parallel to the beam line and provide the track measurement in the magnetic bending plane $(\mathrm{r}, \phi)$. In the central SL, the wires are orthogonal to the beam line and measure the position along the beam direction. The RPCs are double-gap chambers operated in avalanche mode, with copper readout strips oriented along the beam direction [3].

\section{2010-2013 DT and RPC system operations}

On 30th March 2010, LHC started proton-proton collisions at a center-of-mass energy of 7 $\mathrm{TeV}$. Commissioning of the machine through all the year turned into a substantial increase of the maximum instantaneous luminosity, achieving up to $2 \cdot 10^{32} \mathrm{~cm}^{-2} \mathrm{~s}^{-1}$ by the end of 2010. 2011 operations have seen enormous increases in luminosity, such that the entire 2010 dataset was delivered in less than a day. The instantaneous luminosity reached up to $3.5 \cdot 10^{33} \mathrm{~cm}^{-2} \mathrm{~s}^{-1}$, setting a new world record for beam intensity at a hadron collider. In 2012, LHC has been operating at the increased energy of $4 \mathrm{TeV}$ per beam, reaching a maximum of instantaneous luminosity of $6.6 \cdot 10^{33}$ $\mathrm{cm}^{-2} \mathrm{~s}^{-1}$. The results discussed in the following sections are based on all data collected until July 2012.

CMS operation has been remarkably smooth with $92 \%$ of data taking efficiencies. The DT and RPC systems performed extremely well: the contribution to the CMS downtime has been below $1.5 \%$, quite constant every year. More than $98 \%$ of RPC channels and 99\% of DT channels were operational, throughout the full period of operations. The fraction of inactive channels, mainly caused by failure of the electronics located near or inside the chambers, not accessibly since 2009, will be recovered during the long shutdown in 2013 and 2014. The excellent performance demonstrates the robustness of the DT and RPC systems and the reliability of the control and monitoring systems. 


\section{DT performance}

\subsection{DT trigger performance}

The DT local trigger (DTLT) system is based on the mean-timer algorithm and it allows reconstructing tracks from the drift time measurements [4]. LHC is designed to run at a bunch crossing (BX) spacing of $25 \mathrm{~ns}$. For each BX, the DTLT provides up to two trigger segments per chamber in the $\phi$ view and one in the $\theta$ view. To measure the DTLT efficiency, selected events are required to be triggered by the RPC system (without any requirement on the presence of a DT trigger); the presence of a track segment with at least 4 out of 8 hits and associated with the muon track is then required in the same chamber. In addition, the selected segment has to be within the angular acceptance of the DT system. In Fig. 1a the local trigger efficiency as a function of the transverse momentum is shown for 2012 data. The small efficiency drop in MB4 station is due to two known problematic chambers. Fig. 1b shows the trigger efficiency distribution for 2011 and 2012 data. The average efficiencies of $93.5 \%$ and $93.7 \%$, respectively, are in good agreement with the design expectations.
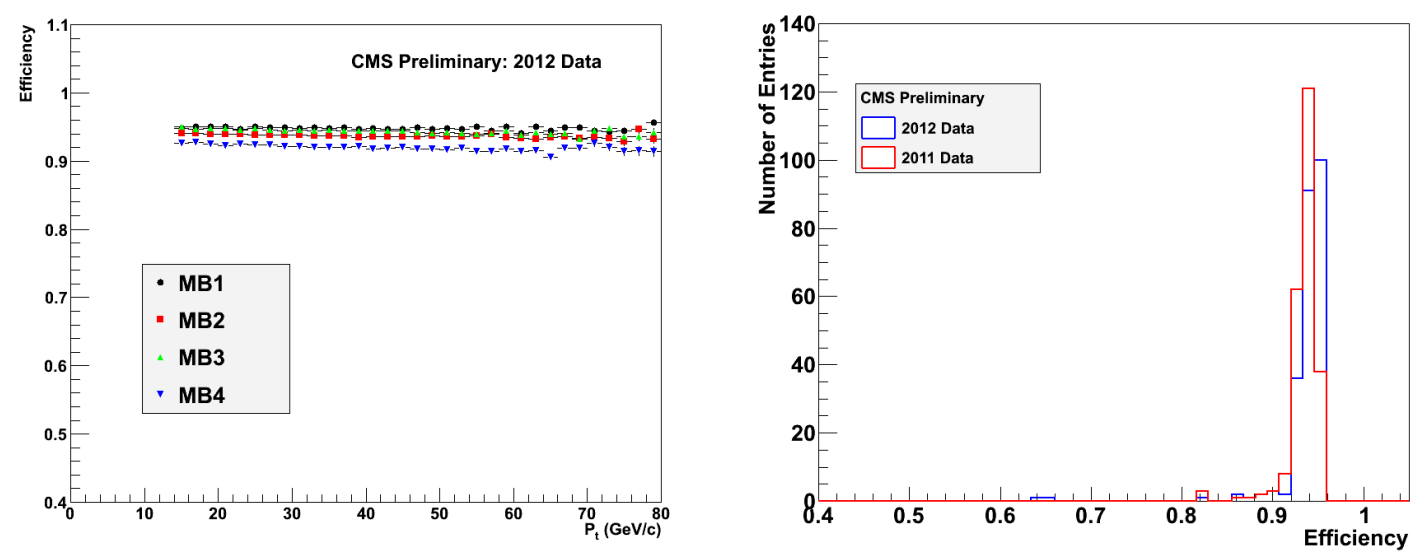

Figure 1: Left: DT local trigger efficiency as a function of the muon transverse momentum for the four barrel stations. Right: DT local trigger efficiency distributions for 2011 and 2012 data.

\subsection{DT local reconstruction efficiency}

The segment reconstruction efficiency was measured using the tag-and-probe method [5] applied to well identified muons from $\mathrm{J} / \psi$ and $\mathrm{Z}$ decays selected from 2011 collision data. The same technique was applied to appropriate samples of simulated events. The overall performance of the DT system is summarized in Fig. 2a, which shows the segment efficiencies for each sector to be better than 97\%. Fig. 2b shows the segment efficiency computed for all barrel sectors and wheels of the MB2 stations as a function of the local $\mathrm{x}$ coordinate (where $\mathrm{x}$ is along the layer, normal to the beams). The observed efficiency matches the Monte Carlo expectations all the way to the edges of the chamber. Similar distributions have been obtained for all other DT stations. 

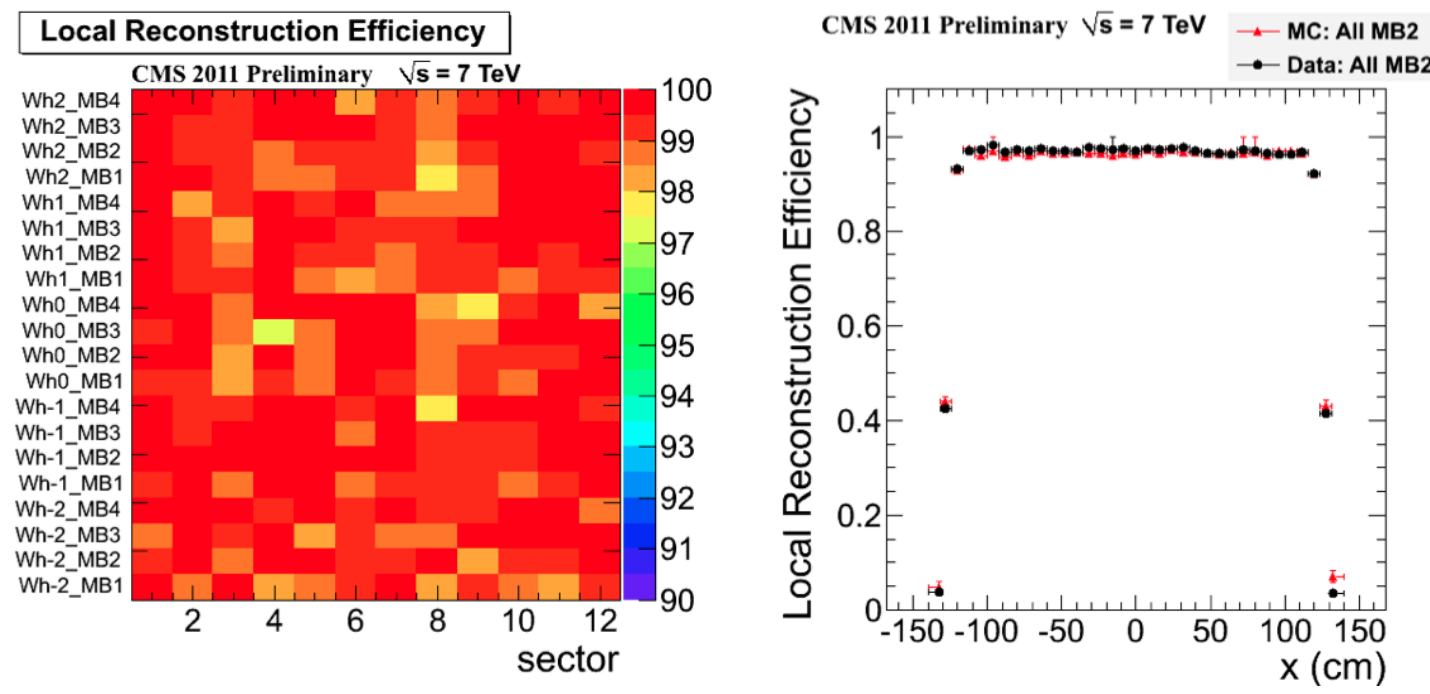

Figure 2: Left: DT segment reconstruction efficiency for all barrel chambers (sectors on the horizontal axis, stations and wheels on the vertical axis). Right: segment reconstruction efficiency for all MB2 stations as a function of local $\mathrm{x}$ (the simulated results are in red).

\section{RPC performance}

\subsection{RPC trigger performance}

The RPC trigger system identifies muons, measures their transverse momenta $\left(\mathrm{p}_{T}\right)$ and selects up to four muon candidates by comparing the pattern of RPC hits in the event to a set of predefined patterns (PAC algorithm) in the same BX. The RPC trigger efficiency was measured using the tag-and-probe method applied to muons from the decays of $\mathrm{J} / \psi$ and $\mathrm{Z}$ resonances to cover a $\mathrm{p}_{T}$ spectrum below and above $20 \mathrm{GeV} / \mathrm{c}$, respectively. Results of the RPC trigger efficiency as a function of the muon $\mathrm{p}_{T}$ from 2011 collision data are shown in Fig. 3. A flat plateau is visible over wide range of $\mathrm{p}_{T}$ with an efficiency value of about $90 \%$.

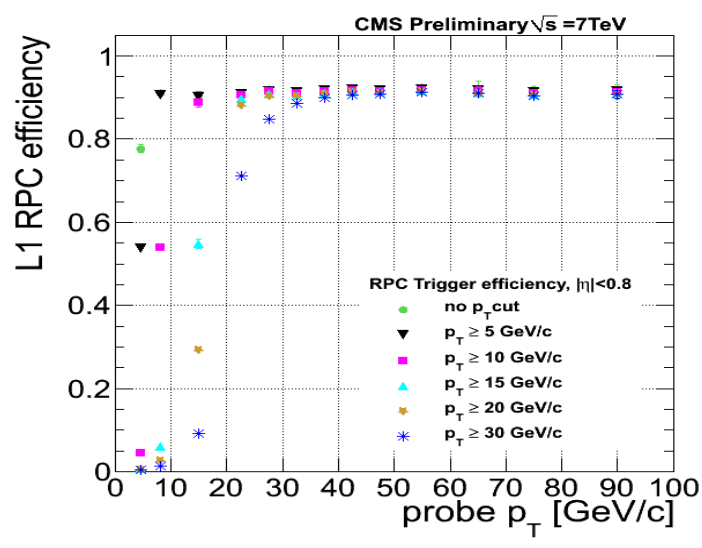

Figure 3: RPC trigger efficiency as a function of the muon $\mathrm{p}_{T}$ from 2011 collision data. 

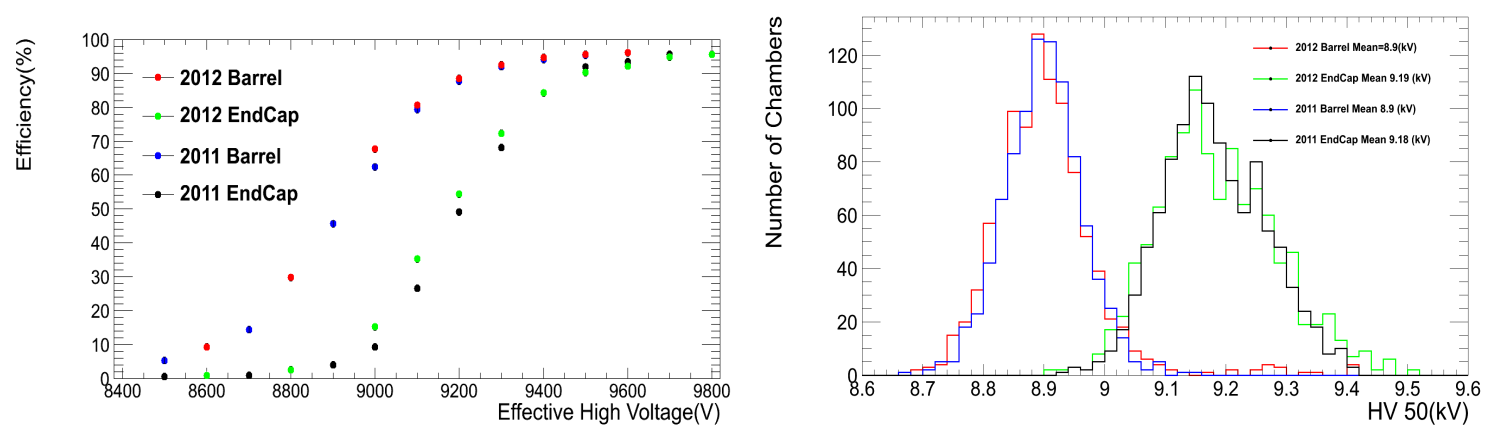

Figure 4: Left: barrel and endcap average efficiency as a function of the $\mathrm{HV}_{e f f}$, measured in 2011 and 2012 HV calibration. Right: the HV distributions for all RPCs as measured at the $50 \%$ of the efficiency.

\subsection{RPC Efficiency}

During 2010, the LHC low luminosity did not allow to tune the working voltage chamber by chamber. Thus only two different values were chosen for operation of the endcap and barrel chambers $(9.5 \mathrm{kV}$ and $9.3 \mathrm{kV}$, respectively). These values were defined on the basis of the measurements performed during the construction and commissioning phases. In early 2011, a High Voltage (HV) calibration was carried out: collision data were recorded at different HV values. Then in early 2012, the HV calibration was repeated to monitor in time the chambers performance and to spot any aging effect. In order to study the chambers efficiency, muon events have been selected, thanks to the redundancy of the muon system, asking for a DT or CSC trigger. Then a linear extrapolation of track segment in DT and CSC chambers was performed toward the closest RPC strip plane and then matched to any RPC cluster in a range of 8 strips around the extrapolated impact point. More details on the method can be found in [6]. Moreover, in order to take into account the atmospheric pressure variations the effective High Voltage $\left(\mathrm{HV}_{e f f}\right)$ was considered [7]. Fig. 4a shows the average efficiency for all barrel and endcap chambers as a function of $\mathrm{HV}_{\text {eff }}$ as measured in 2011 and 2012 HV calibrations. The high voltage distributions as measured at the 50\% of the efficiency is shown in Fig. 4b. No significative variations have been observed on working point and on chamber efficiency plateau values. The chamber performance is monitored in time, run-by-run, in order to study the stability of the system. In Fig. 5 the overall barrel efficiency as a function of run number is shown. A maximum variation of the efficiency of about $3 \%$ was observed in the first three months of 2011 data taking, reduced to $1 \%$, since July once an automatic correction of the HV working point with the atmospheric pressure measured in the CMS cavern was implemented.

\section{Background}

The background radiation levels can have important impact on the aging of the detectors and the overall performance of the system: high background levels could affect the trigger performance and pattern recognition efficiency of muon tracks.

The RPC background rate has been measured by counting the number of hits per second in a single strip, in a fixed time interval of $100 \mathrm{~s}$. Fig. 6a shows the RPC background rate in the 


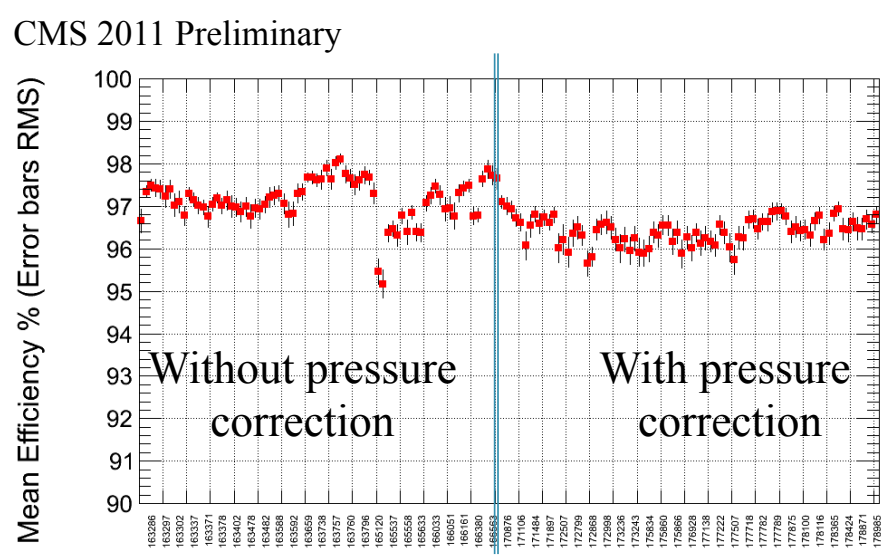

Figure 5: Barrel average efficiency as a function of run number (from April to October 2011). The vertical line separates the runs taken without and with the HV pressure correction.

four YB-2 muon stations as a function of the 2011 LHC instantaneous luminosity. As expected the highest rates are in the outermost station (RB4), mainly due to neutron background, and in the innermost (RB1), mainly affected by particles coming from the vertex [1].

The DT background rate has been measured by requiring only 1-2 hits within a SL (in comparison with the 3-4 hits obtained from a muon-like track), integrating over the full $1.25 \mu$ s read-out window. Fig. $6 \mathrm{~b}$ shows the DT background rate in the innermost station MB1 of the wheels as a function of the 2012 LHC instantaneous luminosity. As expected the rates increase with distance from the Interaction Point (YB0 is closest to the IP, $\mathrm{YB} \pm 2$ are farthest away). The different RPC and DT background values are due to their different gamma and neutron sensitivity.

Based on the previous results a linear extrapolation to the LHC luminosity of $10^{34} \mathrm{~cm}^{-2} \mathrm{~s}^{-1}$ is attempted. The highest expected RPC background rate is $\sim 35-40 \mathrm{~Hz} / \mathrm{cm}^{2}$ and $\sim 15-20 \mathrm{~Hz} / \mathrm{cm}^{2}$ in the endcap and barrel region, respectively, well below the limit of $100 \mathrm{~Hz} / \mathrm{cm}^{2}$ used in the RPC trigger design. Less than $\sim 5 \mathrm{~Hz} / \mathrm{cm}^{2}$ is the expected DT maximum background value.
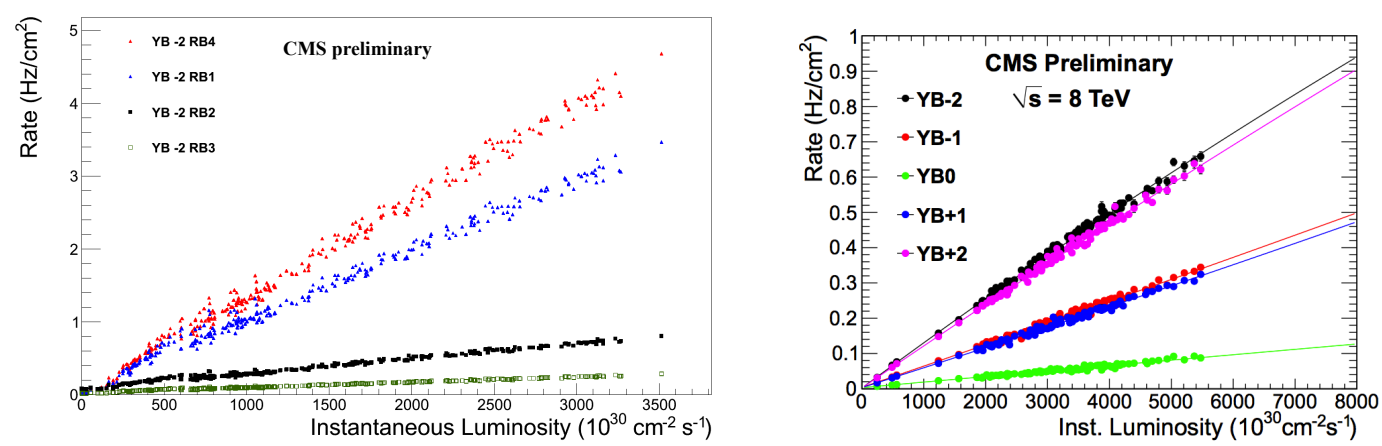

Figure 6: Left: The RPC backgrounds rate vs. 2011 instantaneous luminosity for the YB-2 wheel. Right: The MB1 DT background rates vs. 2012 LHC instantaneous luminosity for the wheels (YB0 is closest to the IP, YB \pm 2 are farthest away). 


\section{Conclusion}

Operation of the CMS DT and RPC muon systems during the first three years of LHC data taking has been outstanding: the contribution to CMS downtime has been below $1.5 \%$ and more than $98 \%$ of RPC and $99 \%$ of DT channels are operational. The DT performance has been studied and it is in agreement with the design expectation: the efficiency for reconstructing local segments is above $97 \%$ and the trigger segments efficiency is on average $93.7 \%$. The RPC operating HV values have been optimized and an automatic HV pressure correction has been applied. The monitoring in time of the RPC efficiencies shows an average efficiency above 96\%, stable within $1 \%$ of variation. The expected background rate at LHC luminosity of $10^{34} \mathrm{~cm}^{-2} \mathrm{~s}^{-1}$ will be sustainable for the present DT and RPC systems.

\section{References}

[1] CMS Collaboration, The CMS experiment at the CERN LHC, J. Instrum. 3 (2008) S08004 doi:10.1088/1748-0221/3/08/S08004

[2] L. Evans and P. Bryant, LHC machine, J. Instrum. 3 (2008) S08001 doi:10.1088/1748-0221/3/08/S08001

[3] CMS Collaboration, The Muon Project Technical Design Report, CERN/LHCC 97-32 (1997)

[4] RD5 Collaboration, Bunch crossing identification at LHC using a mean-timer technique, Nucl. Instr. Methods Phys. Res. A, 336 (1993) 91

[5] CMS Collaboration, Performance of CMS muon reconstruction in pp collision event at $\sqrt{s}=7 \mathrm{TeV}$, JINST 7 (2012) P10002, doi:10.1088/1748-0221/7/10/P10002,

[6] CMS Collaboration, Calibration of the RPC working voltage in the CMS experiment at the LHC, JINST 7 (2012)

[7] M. Abbrescia, et al., Resistive plate chambers performances at cosmic rays fluxes, Nucl. Instr. Methods Phys. Res. A, 359 (1995) 603 